\title{
Reading and Mild Cognitive Impairment
}

\author{
Kristina Lundholm Fors ${ }^{1}$, Malin Antonsson ${ }^{1}$, Dimitrios Kokkinakis ${ }^{1,2}$, \\ Kathleen C. Fraser ${ }^{3}$ \\ ${ }^{1}$ Department of Swedish, University of Gothenburg, Sweden \\ ${ }^{2}$ Centre for Ageing and Health, University of Gothenburg, Sweden \\ ${ }^{3}$ Digital Technologies Research Centre, National Research Council Canada, Canada \\ https://doi.org/10.36505/ExLing-2019/10/0021/000383
}

\begin{abstract}
In the present study, we investigated the discriminatory power of eye-tracking features in distinguishing between individuals with mild cognitive impairment (MCI) and healthy controls (HC). The eye movements of the study participants were recorded at two different time points, 18 months apart. Using a machine learning approach with leaveone-out cross-validation, we were able to discriminate between the groups with 73.6 AUC. However, somewhat surprisingly the classification was less successful using data from the second recording session, which might be attributed to the non-static nature of cognitive status. Still, the outcome suggests that eye-tracking measures can be exploited as useful markers of MCI.
\end{abstract}

Key words: mild cognitive impairment, dementia, eye-tracking, reading.

\section{Introduction and background}

Dementia is a neurodegenerative disorder that is becoming more prevalent as the global population ages. In order to provide the best care and enable the alleviation of symptoms through lifestyle changes and access to medication, it is important to identify individuals at risk for dementia at an early stage. In this study, we used eye-tracking while reading as a means to identify persons with mild cognitive impairment (MCI). MCI is defined as a mild state of cognitive decline, which can affect several cognitive domains such as episodic memory, executive functioning and linguistic ability, and persons with MCI are at higher risk of developing dementia (Gauthier et al. 2006). As reading processes are dependent on several cognitive processes related to language and short-term memory, we believe that capturing the reading process with eye-tracking will allow us to differentiate between persons with MCI and healthy controls. Previous work has shown the effectiveness of using eye-tracking to detect signs of dementia due to Alzheimer's disease (Lueck, Mendez, Perryman 2000, Fernández et al. 2013, Biondi, Fernandez, Castro, Agamenonni 2017), but very little work has examined the potential utility of this technology for detecting milder phases of impairment (Fraser, Lundholm Fors, Kokkinakis, Nordlund 2017). In this study, we examine the performance of machine learning classifiers trained on eye movements collected at two different time points, on the task of discriminating between participants with MCI and healthy controls.

ExLing 2019: Proceedings of $10^{\text {th }}$ International Conference of Experimental Linguistics, 25-27 September 2019, Lisbon, Portugal 


\section{Method \\ Participants}

The participants in this study are a subcohort of the study population reported in Kokkinakis, Lundholm Fors, Björkner \& Nordlund, (2017), which describes a broader project on language-related markers of early-stage cognitive impairment. In the current analysis, we include only those participants who successfully completed the eye-tracking component of the protocol at two different time points; specifically, 15 participants with MCI and 23 healthy controls (HC). Near the time of the first data collection session, each participant underwent a detailed neuropsychological assessment. The groups differed significantly on the MMSE (a test of cognitive functioning with a maximum of 30; MCI 28.6, HC 29.7), but not on age (mean age in years: MCI: 71.3, HC: 67.6) or education (mean education in years: MCI 14.0, HC 13.7). All participants were native speakers of Swedish. The study was approved by the local ethical committee review board (reference number: 206-16, 2016).

\section{Data}

The task consisted of reading two short texts (one silently and one aloud) while eye movements were recorded with an EyeLink 1000 Desktop Mount (sampling rate: $1000 \mathrm{~Hz}$ ). The participants performed the same task twice, with 18 months in between. The eye-tracking data were analysed with a focus on several aspects of reading: fixations, saccades, reading duration etc. Each reading sample was represented by thirteen features, as proposed by Biondi et al. (2017) and further described by Fraser et al. (2017).

\section{Statistical analysis and machine learning classification}

To test whether the eye-tracking data could be used to detect MCI, a machine learning experiment was performed. We compared three different classification algorithms: naïve Bayes (NB), support vector machine (SVM) with RBF kernel, and logistic regression (LR) with L2 regularization, all implemented in Python using the Scikit-learn library (Pedregosa et al., 2011). Each classifier was evaluated using leave-one-out cross-validation, with area under the ROC curve (AUC) as the evaluation metric. Features were standardized according to the training set in each fold, and default hyper-parameters were used. Additionally, we performed two-tailed t-tests with Bonferroni corrections for multiple comparisons, in order to investigate which features differed the most between the MCI group and the HC.

\section{Results}

The classification results (Table 1) are consistently better for Session 1 (i.e. the first recording), with the best result of 0.736 compared to 0.658 for Session 2. Statistically, no difference in either session was significant at the corrected level $(\mathrm{p}<0.004)$. However, in Session 1, two features differ on the $\mathrm{p}<0.05$ level: the 
MCI group has a larger number of "unique" fixations (fixations that occur once on words that were skipped in the first pass $)(p=0.014)$, and the standard deviation of the duration of the first pass fixations is greater in the MCI group $(p=0.023)$. In Session 2, one feature differs on the $p<0.05$ level: the persons with MCI have a greater mean amplitude of saccades than the controls $(p=0.046)$.

Table 1: Machine learning classification results (AUC).

\begin{tabular}{|l|l|l|l|}
\hline \hline & Logistic Regression & SVM & Naïve Bayes \\
\hline Session 1 & 0.713 & 0.736 & 0.728 \\
\hline Session 2 & 0.644 & 0.524 & 0.658 \\
\hline \hline
\end{tabular}

\section{Discussion}

The classification results from Session 1 and Session 2 in this study show that classification accuracy decreases from Session 1 to Session 2, even though we use data from the same participants. This is somewhat surprising, as we would expect differences between the MCI group and HC group to increase over time, as cognitive deterioration progresses. However, previous longitudinal studies report that up to $44 \%$ of persons fulfilling the criteria for MCI may return to normal within a year (Gauthier et al., 2006). Conversely, the prevalence of MCI increases with age, which would suggest that with time, the HC group is more at risk of developing MCI.

While no features were significant after correcting for multiple comparisons, the features that differ significantly on the $p=0.05$ level can be interpreted in the context of MCI. The tendency in the MCI group to skip words in the first pass and come back to them in a later pass is consistent with them producing a greater number of "unique" fixations (fixations that occur once on words that were skipped in the first pass), and the greater mean amplitude of saccades also suggests that the MCI group make larger movements within the text instead of moving consistently from one word to the next. This disruption in the reading process can be attributed to the impairments in short term memory and executive function seen in MCI. The feature analysis also implies that there is a greater amount of variability in fixation durations in the MCI group, suggesting a more irregular pace through the text. These findings are consistent with our previous work (Fraser et al., 2017), in which we concluded that the MCI and HC groups differed significantly in the linearity of the reading process.

In sum, this study highlights how eye-movement data from a longitudinal MCI-study can be used for classification purposes. Although the decrease in classification accuracy at first seemed surprising, this might be explained by variability in the cognitive status in both groups of participants, and underscores the need for updated neuropsychological assessments when evaluating persons with MCI longitudinally. The participants in our study are currently undergoing renewed neuropsychological assessments, and in future 
work, we plan to incorporate these results to evaluate if they explain the variation in the results, and if they can be used for better prediction modelling.

\section{Acknowledgements}

This work has received support from The Swedish Foundation for Humanities and Social Sciences (grant agreement no: NHS 14-1761:1).

\section{References}

Biondi, J., Fernandez, G., Castro, S., Agamenonni, O. 2017. Eye-Movement behavior identification for AD diagnosis. arXiv preprint arXiv:1702.00837.

Fernández, G., Mandolesi, P., Rotstein, N.P., Colombo, O., Agamennoni, O., Politi, L.E. 2013. Eye movement alterations during reading in patients with early Alzheimer disease. Investig. Ophthalmol. Vis. Sci. 54, 8345-8352.

Fraser, K.C., Lundholm Fors, K., Kokkinakis, D., Nordlund A. 2017. An analysis of eye-movements during reading for the detection of mild cognitive impairment. Proceedings of the 2017 Conference on Empirical Methods in Natural Language Processing, 1016-1026.

Gauthier, S., Reisberg, B., Zaudig, M., Petersen, R., Ritchie, K., Broich, K., Winblad, B. 2006. Mild cognitive impairment. Lancet 367, 1262-1270.

Kokkinakis, D., Lundholm Fors, K., Björkner, E., Nordlund, A. 2017. Data collection from persons with mild forms of cognitive impairment and healthy controls infrastructure for classification and prediction of dementia. Proceedings of NoDaLiDa. 131; LiU Electronic Press;172-82.

Lueck, K.L., Mendez, M.F., Perryman, K.M. 2000. Eye movement abnormalities during reading in patients with Alzheimer disease. Neuropsychiatry, Neuropsychology, \& Behavioral Neurology.

Pedregosa, F., Varoquaux, G., Gramfort, A., Michel, V., Thirion, B., Grisel, O., Duchesnay, E. 2011. Scikit-learn: Machine Learning in Python. Journal of Machine Learning Research 12, 2825-2830. 